\title{
A RARE HEPATIC-VENOUS ANOMALY WITH PORTAL DRAINAGE OF THE PULMONARY VEINS
}

\author{
BY \\ JAMES B. GIBSON AND C. A. J. MACAFEE \\ From the Department of Pathology, Queen's University, Belfast \\ Received August 11, 1960
}

Major congenital defects of the hepatic veins are readily detectable at autopsy but they have been described rarely. In the case reported here an anomalous disposition of these veins brought venous blood from the left half of the liver into the left atrium and blood from the right half of the liver into the right atrium. We have traced only two other similar cases (Arnold, 1868; Epstein, 1886).

Pulmonary venous anomalies are better documented. They may divert the whole or a part of the pulmonary venous return from the left atrium into the right atrium or its tributaries. We shall neglect anomalies of parts of the pulmonary veins, for the present case is one of total anomalous venous drainage. Brody (1942) first subdivided this condition into an isolated form accompanied only by a patent foramen ovale or ductus arteriosus which makes the circulation possible, and into a complicated form in which the lesion is associated with major congenital cardiac defects, as in our case. Keith et al. (1958) recorded the insertions of the common pulmonary vein in 103 cases of the isolated form of the disease: in 11 of these the insertion was into the portal vein or the ductus venosus. Edwards (1960) stated that 66 cases of the complicated form of total anomalous pulmonary venous drainage were to be found, mostly reported cases but some in the material of the Mayo Clinic. The associated cardiac defects have included cor biloculare and truncus arteriosus: persistent atrio-ventricular canal, gross systemic venous malformations, and agenesis of the spleen, which were found in the present case, have also been recorded. Darling et al. (1957) registered infra-diaphragmatic insertions of the anomalous pulmonary veins in 10 out of 40 descriptions of the complicated form that they studied. Butler (1952) has divided cases of both forms of portal drainage of the pulmonary veins into group A in which the ductus venosus is the recipient vessel, as in our case, and group B in which the common pulmonary vein enters the left gastric vein.

\section{CASE REPORT}

A male infant was born normally at term to a primigravida of 25 , who had suffered from no illness during the pregnancy. The infant breathed poorly, there was peripheral cyanosis, and the tone was limp. Air entry into the right lung was poor and the right side of the chest was drawn in on inspiration. A short systolic murmur was heard over the xiphisternum. The cyanosis deepened on exertion. The infant's condition deteriorated progressively; a convulsion and a hæmatemesis occurred on the morning of the third day and the infant died shortly thereafter.

\section{Necropsy}

The body weighed $3750 \mathrm{~g}$. There was slight œdema of the face only and the xiphisternum was prominent. There were no effusions into the body cavities. Most of the abnormal findings, except those of the pulmonary veins, are represented diagrammatically in Fig. 1.

The heart lay in the normal position and was not enlarged. The pulmonary trunk arose from the right ventricle through the pulmonary valve, which was $4 \mathrm{~mm}$. in diameter and bicuspid, and divided normally into branches for each lung. The ductus arteriosus, which joined the right pulmonary artery to the right 
aortic arch, was closed. The aorta lay posterior to the pulmonary trunk and was separated from the pulmonary valve by a band of muscle $3 \mathrm{~mm}$. high (crista supraventricularis). The aorta arched to the right, giving off the left innominate, right common carotid, and right subclavian arteries. The aorta was partially dextraposed and gave origin to the two coronary arteries. The aortic valve had three cusps.

Inside the heart there was a defect $2 \mathrm{~cm}$. in diameter in the lower part of the atrial septum and the upper part of the ventricular septum. The valve of this common atrio-ventricular canal was formed by anterior and posterior cusps which were each continuous through the septal defect and were attached to chordæ tendinex of both ventricles. The defective portion of the ventricular septum lay below the line of closure of the cusps so that the ventricles communicated in a subvalvular space. Part of the atrial septum was

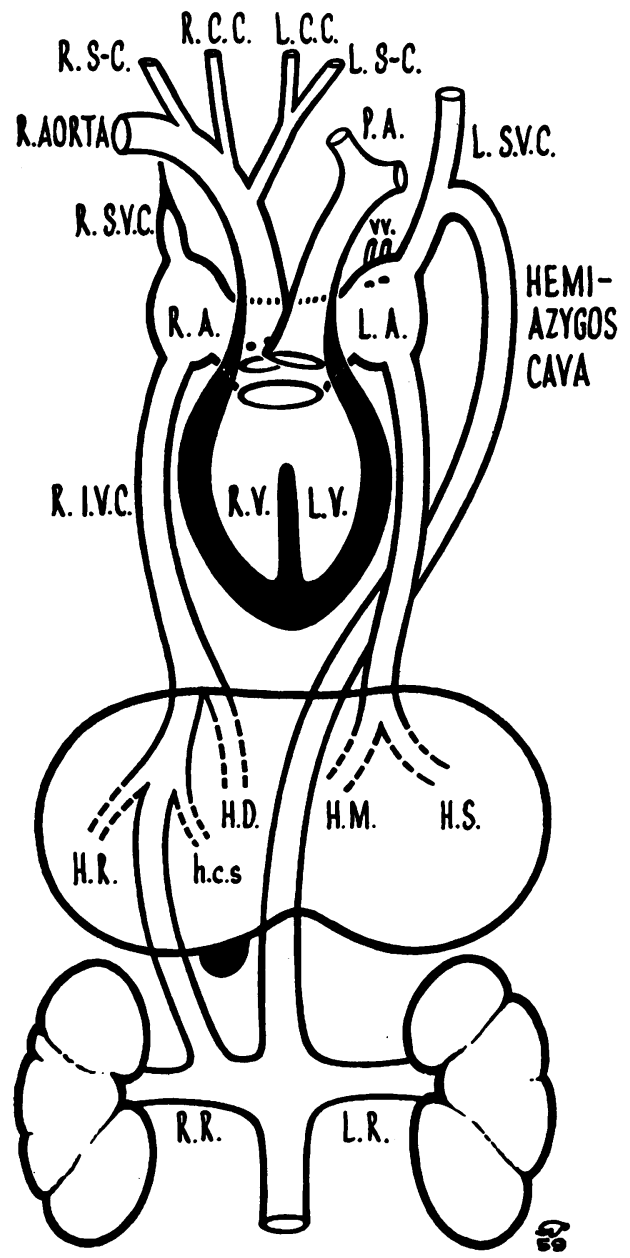

FIG. 1.-Diagram of the systemic venous and cardiac anomalies.

$\mathbf{R R}$, right renal vein.

LR, left renal vein.

HR, vena hepatica dorsalis.

$H D$, vena hepatica dextra.

HM, vena hepatica media.

HS, vena hepatica sinistra.

hcs, venula hepatica caudata superior.

RIVC, right inferior vena cava.

RA, right atrium.

LA, left atrium.
RV, right ventricle.

$\mathrm{LV}$, left ventricle.

vv, minute cardiac veins.

RSVC, atretic right superior vena cava.

LSVC, left superior vena cava.

PA, pulmonary artery.

$R$ and LSC, right and left subclavian arteries.

$R$ and LCC, right and left common carotid arteries.

present above the interatrial defect but it was fenestrated. This form of common atrio-ventricular canal has been defined as the complete type by Wakai and Edwards (1958).

Venous Anomalies. Into the right atrium ran a minute and atretic vessel representing the right superior vena cava. The main vessel entering the right atrium was a right inferior vena cava. It began as a branch of the right renal vein and, passing upwards, ran for $2 \mathrm{~cm}$. in a groove on the postero-lateral surface of the liver. Next it occupied the normal site of the hepatic part of the inferior vena cava, i.e. between caudate and right lobes and received hepatic veins from the caudate lobe (hcs) and the right lobe (HR, HD) as usual near the diaphragm. The left hepatic ostium was not present.

The lower part of the inferior vena cava was normal, but above the junction of the renal veins, it failed 
to establish the usual direct connection with the hepatic portion. Instead it ran leftwards and upwards, lying posterior to and to the left of the right inferior vena cava, and entered the thorax in the position of the inferior hemiazygos vein. This large vessel, which has been designated the hemiazygos cava, finally joined a left superior vena cava to enter the left atrium as a persistent left duct of Cuvier. A few minute veins entered the left atrium close to the duct of Cuvier and apparently represented the oblique vein and the coronary sinus.

A second large vein entered the lower part of the left atrium. This left common hepatic vein arose in the liver but was entirely separate from the hepatic part of the right inferior vena cava and lay to the left of it. It was made up of the two veins (HS and HM), which normally unite to form the left ostium, and thus drained the left lobe and central mass of the liver. A connection could not be traced between this vessel and the ductus venosus.

The pulmonary veins were not connected in any way to either atrium but united in a $\mathrm{T}$ junction to form a large common pulmonary vein (Fig. 2). This ran downwards, posterior to the vessels described above,
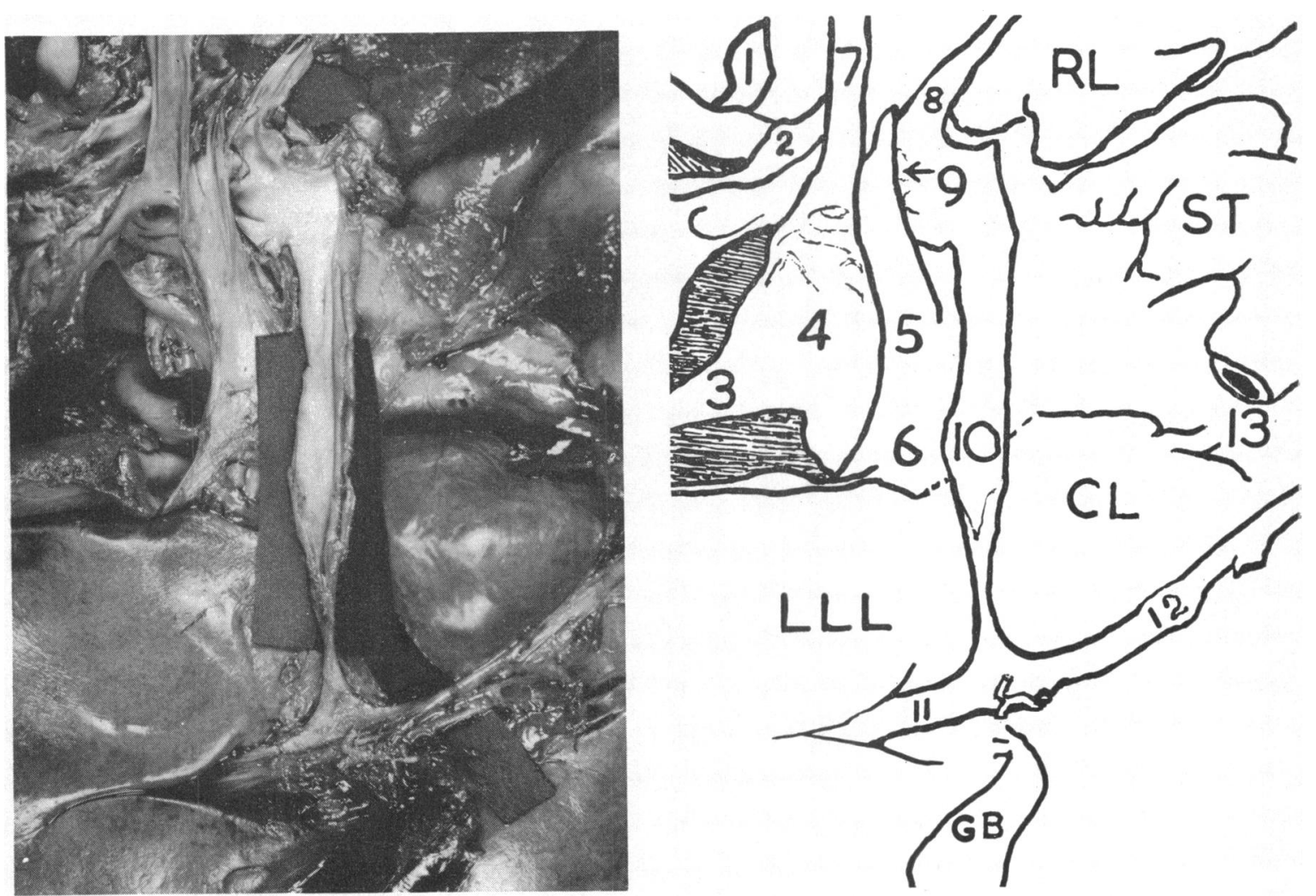

FIG. 2.-Posterior view of the viscera to show common pulmonary vein narrowing before entering portal sinus. The stomach has been turned upwards and to the right. Other structures are labelled in the outline drawing as follows.

1. aortic arch.

2. pulmonary artery.

3. cavity of left ventricle.

4. cavity of left atrium.

5. posterior wall of left atrium.

6. entry of left common hepatic vein into left atrium.
7. left superior vena cava.

8. right pulmonary vein.

9. left pulmonary vein.

10. common pulmonary vein.

11. left branch of portal vein.

12. portal sinus.
13. right inferior vena cava with

RL, right lung.

LLL, left lobe of liver.

ST, stomach.

$\mathrm{CL}$, caudate lobe.

$\mathrm{GB}$, gall bladder.

reaching the groove for the ductus venosus to the left of the caudate lobe, finally to enter the left part of the portal sinus in the place of the ductus with which it was apparently incorporated. Towards its termination the common pulmonary vein was narrowed (Fig. 2), and histological sections showed muscular contraction 
and intimal fibrosis like that seen in the physiological obliteration of the ductus venosus. The sections confirmed the absence of any other structure in this region corresponding to the ductus venosus.

Obliteration of the umbilical vein had begun. The spleen was absent and there was no trace of a splenic artery or vein. No other anomalies of the portal venous system were found. The liver was of the usual shape and not abnormally lobate. The gut was normally rotated. The stomach did not contain any blood, and varices were not seen on gross or microscopical examination of the œsophagus. The structure of the lungs appeared normal on gross and on microscopical examination. In the rest of the post-mortem examination there were no other abnormal findings.

\section{DiscUSSION}

The prognosis in cases of anomalous pulmonary venous drainage diagnosed during life is not uniformly bad, but Keith et al. (1958) found that in the isolated form of the anomaly, an infradiaphragmatic insertion of the pulmonary vein carried a bad prognosis, even in infancy. Butler (1952) noted that, in cases of both isolated and complicated forms, survival was shorter when the pulmonary vein entered the ductus venosus (group A) than when it was inserted into the left gastric vein (group B). He pointed out that the obliteration of the ductus venosus in the neonatal period is likely to have a more deleterious effect in group A than in group B cases. The gross narrowing of the common termination of the pulmonary vein and the ductus venosus in our case (Fig. 2) was probably due to this physiological process. It is considered to have been the cause of death, for the ductus was not connected superiorly with any hepatic or other vein, and obliteration of the lower end sealed the only demonstrable outlet for venous blood from the lungs. Laurence and Brown (1960) have reported massive and fatal hæmatemesis from œsophageal varices in a baby with the isolated form of the anomaly and insertion of the common pulmonary vein into the left gastric vein. Hæmatemesis was recorded terminally in our patient's history, but the bleeding was small and its origin was not located. Varices were not present in the œsophagus and the left gastric vein was not dilated.

Duckworth (1958) dates the pathogenesis of anomalous venous drainage of the lungs between the third and eighth weeks of embryonic life. The failure of the pulmonary veins to join the left atrium may be regarded primarily as a cardiac malformation that in turn necessitates the retention of more primitive channels of venous drainage from the lungs. Butler (1952) cites the evidence for the view that the cardio-pulmonary venous junction is normally effected between an outgrowth of the dorsal wall of the sinu-atrial chamber and the pulmonary portion of the foregut venous plexus. When this new outflow into the left atrium is not achieved, a primitive interconnection of the pulmonary part of the foregut venous plexus with the œsophageal or gastric part may persist and give rise to a common pulmonary vein with insertion into the left gastric vein. Butler supports this view of the origin of the type B cases by referring to the persistence in normal rats of portapulmonary venous connections along the vagus nerves, and refers to comparable veins in normal adult men. Venous shunting through such veins in cirrhosis may be responsible for undersaturation of arterial blood with oxygen and so for finger clubbing in that condition (Georg et al., 1960). Butler considers that connections of the common pulmonary vein with the ductus venosus in type A cases are likewise persistent elements of the foregut venous plexus. This view is more difficult to substantiate because there are no parallels to be drawn from normal human or comparative anatomy, and because the liver, which originates from the caudal part of the foregut, derives its venous supply from the vitelline veins and not from the true foregut plexus. Between these two venous beds, however, there are connections that may take part in the early formation of the ductus venosus. The ductus venosus develops as an integral part of the venous system of the liver, and so from the vitelline veins and not from the left umbilical vein (Huzly, 1942; Dickson, 1957), as was once supposed.

The upper part of the ductus venosus was absent in our case (Fig. 2) and this may be related to the anomalies of the hepatic veins. At the 5-mm. stage (fourth-fifth weeks) the liver is drained by the primary right and primary left hepatic veins into the respective ducts of Cuvier (common cardinal 
veins) close to the sinus venosus (Dickson, 1957). The veins are united by the subdiaphragmatic anastomosis which receives the upper end of the ductus venosus. Normally during the ensuing week the primary left hepatic vein loses its connection with the left side of the heart and draws over to the right, absorbing the subdiaphragmatic anastomosis together with the attachment of the ductus venosus, and becomes the secondary left hepatic vein. This definitive vessel unites proximally with the right hepatic vein, which has not undergone any comparable metamorphosis, to form the common hepatic vein, which drains into the right atrium and gives origin to the hepatic portion of the inferior vena cava. The absence of an upper attachment of the ductus venosus in our case is explained by the failure of the secondary left hepatic veins and possibly of the subdiaphragmatic anastomosis to develop at all. The hepatic veins from the median mass (HM) and from the left lobe (HS) emptied into a common left hepatic vein or persistent primary left hepatic vein, which maintained its drainage into the left atrium (Fig. 1). The malformation may be regarded as essentially a failure in closure of the sinu-atrial mouth of the primary left hepatic vein. As with the pulmonary venous anomaly, we are led to suspect a primary defect in the left side of the sinuatrial chamber about the fifth week.

Other Reported Cases. A case reported by Arnold (1868) resembles ours in several respects although it was apparently complicated by situs inversus of the heart. Arnold described a single ventricle, a single ventricular efferent valve, and an atrial septal defect. A common pulmonary vein ran parallel to the ductus venosus and discharged by three channels into separate portal venous branches in the porta hepatis. The spleen was absent. The inferior vena cava lay in a groove on the left side of the liver, and was joined by the left hepatic vein (HS) and by some other small veins before entering the left (systemic) atrium. The superior vena cava and coronary sinus also entered the left atrium. The right atrium received a common hepatic vein of which the radicles were the right and median hepatic veins (HD and HM). Thus both atria received hepatic veins as in our case. We have traced only one other report of drainage of hepatic venous blood into both right and left atria. Epstein (1886) described an 11-day-old infant with the complicated form of total anomalous pulmonary venous drainage and absence of the spleen. The common pulmonary vein joined the superior vena cava which entered the right atrium. Some of the hepatic veins also drained via systemic veins into the right atrium, for part of the hepatic venous outflow entered the inferior vena cava directly and blood from an anomalous lobe of liver reached the cava indirectly via an abnormal left renal vein. The greater part of the hepatic venous blood, however, entered the left atrium directly.

In other cases of the complicated form of anomalous pulmonary venous drainage, the hepatic portion of the inferior vena cava has been absent and the hepatic veins have united in a single trunk to join the heart directly. The anomalous pulmonary venous drainage was total in the case of Schelenz (1909) and partial in the case of Lawrence and Nabarro (1901-02) in which the spleen was absent. Other anomalies of the hepatic veins have been reported in cases in which the pulmonary veins had developed normally. In the case of Griffith (1891-92), situs inversus and what was apparently a persistent atrio-ventricular canal were found together with absence of the ductus venosus and of the hepatic portion of the cava down to the renal veins; the liver consisted of four separate lobes which the author named right, left, spigelian, and quadrate lobes, but the blood from their veins entered the systemic atrium. In Nabarro's (1902-03) second case a common left hepatic vein, similar in composition to that in the present case, joined a left superior vena cava together with the coronary sinus and so passed to the right atrium. In this case of Nabarro's, in contrast with ours, the definitive left hepatic vein failed to preserve the primitive insertion of the primary left hepatic vein.

Other malformations of the hepatic veins are secondary to anomalies of the portal vein (Hellweg, 1954) or of the inferior vena cava (Abernethy, 1793; Miller, 1925; Huseby and Boyden, 1941; and Effler et al., 1951). We have recently examined at autopsy a 5-week-old infant in which the hepatic portion of the cava, complete with hepatic venous ostia, was united inferiorly to a persistent left inferior vena cava emptying into the right atrium. Other congenital anomalies were present in 
the inferior vena cava, and the ductus venosus was absent; the stomach, duodenum, and spleen, which was composed of several spleniculi, were transposed and there was a pulmonary infundibular stenosis of the heart. Schelenz (1909) reviewed some comparable cases.

Anomalies of the inferior vena cava have been described by other authors. Edwards (1951) has suggested a classification for some of them. The embryological development of the caval system is summarized by Hamilton et al. (1952) who refer to some of the original papers. The right inferior vena cava in our case received veins from the right half only of the liver (Fig. 1). The hepatic cava was defective, not only in the absence of a left ostium but also in a proper connection with the lower part of the cava. Normally a caudal protrusion of the hepatic cava joins the intersubcardial anastomosis to form the portion of the cava between the liver and the renal veins. The fact that this portion was entirely wanting may possibly be associated with the failure of the left hepatic venous elements to join the hepatic portion of the cava and may thus be related to the other anomalies in the case. The hepatic cava was abnormally connected to the right renal vein through a vessel occupying the course of the right ascending lumbar vein, a derivative of the embryonic medial sympathetic vein of the azygos line system.

The main outflow from the inferior vena cava took another course, and reached the left superior vena cava through a vein that was probably derived from the left medial sympathetic vein and that we have named the hemiazygos cava (Fig. 1). Atrophy of the left superior vena cava normally begins about the seventh week with increasing predominance of the right superior vena cava and with transfer of its cardiac tributaries, the oblique vein and the coronary sinus, to the right atrium. A few small veins entering the left atrium in our case were taken to be the cardiac veins, so that the whole complex of the left duct of Cuvier had remained persistently patent and attached to the left side of the heart. The right superior vena cava never assumed its proper importance and was atretic. Perhaps this persistence of the embryonic state was due, like the other venous anomalies in the case, to some defect present in the left part of the sinu-atrial chamber at the fifth week.

The relationship of the splenic agenesis to the venous anomalies in our case is not clear, but the spleen has been reported to be absent in various instances of situs inversus, of congenital heart disease, and of venous anomalies. Jaffe (1921) published a case of splenic agenesis which was associated with persistent left duct of Cuvier, persistent left anterior cardinal vein, and absence of the hepatic portion of the cava: in the heart there was a persistent common atrio-ventricular canal. This form of cardiac malformation is rarely associated with congenital venous anomalies (Rogers and Edwards, 1948; Wakai and Edwards, 1958), but the association occurred in our case and in the case of Griffith (1891-92), as well as in that of Jaffe. Common atrio-ventricular canal results from failure of the endocardial cushions and the atrial septum primum to unite. This fusion normally occurs about the sixth week of intrauterine life (Duckworth, 1958). Thus persistent atrio-ventricular canal is determined at the fifth week, the period at which we have postulated a basic defect in the left sinu-atrial cavity as the origin of the venous anomalies.

\section{SUMMARY}

At autopsy on a 3-day-old infant the pulmonary veins were found to drain entirely into the portal sinus at a site corresponding to the lower end of the ductus venosus. Death was due to narrowing of this common pulmonary vein by a process resembling the physiological obliteration of the ductus venosus.

A common atrio-ventricular canal persisted in the heart, the aorta was partially dextraposed, and the aortic arch was right sided. The right atrium received an atretic right superior vena cava and a right inferior vena cava that arose from the right renal vein. Blood from the right half of the liver drained into the right inferior vena cava and so into the right atrium. The left half of the liver was served by a left common hepatic vein which entered the left atrium directly. The left atrium also received a persistent duct of Cuvier composed of a left superior vena cava and a hemiazygos cava which was continuous with the lower part of the inferior vena cava. The spleen was absent. 
The case is compared with other examples of total anomalous pulmonary venous drainage and of congenital defects of the hepatic veins. Hepatic venous drainage into both atria is exceedingly rare.

The embryological basis of the anomalies is briefly considered. A defect in the region of the left sinu-atrial cavity about the fifth week of intrauterine life is postulated as a possible precursor of most of the anomalies.

We are indebted to Professor F. M. B. Allen for the clinical history, to Mr. G. Smith for the drawing of Fig. 1, and to Mr. D. McA. Mehaffey for the photograph.

\section{REFERENCES}

Abernethy, J. (1793). Philos. Trans., 83, 59.

Arnold, J. (1868). Virchow's Arch., 42, 449.

Brody, H. (1942). A.M.A. Arch. Path., 33, 221.

Butler, H. (1952). Thorax, 7, 249.

Darling, R. C., Rothney, W. B., and Craig, J. M. (1957). Lab. Invest., 6, 44.

Dickson, A. D. (1957). J. Anat., Lond., 91, 358.

Duckworth, J. W. A. (1958). In Heart Disease in Infancy and Childhood, by Keith, J. D., Rowe, R. D., and Vlad, P. (1958) pp. $106 \mathrm{ff}$.

Edwards, E. A. (1951). Angiology, 2, 85.

Edwards, J. E. (1960). In Pathology of the Heart, ed. Gould, S. E. Charles C. Thomas, Springfield, Ill., p. 260.

Effler, D. B., Greer, A. E., and Sifers, E. C. (1951). J. Amer. med. Ass., 146, 1321.

Epstein, A. (1886). Z. Heilk., 7, 308 .

Georg J., Mellemgaard, K., Tygstrup, N., and Winkler, K. (1960). Lancet, 1, 852.

Griffith, T. W. (1891-92). J. Anat., Lond., 26, 117.

Hamilton, W. J., Boyd, J. D., and Mossman, H. W. (1952). Human Embryology. W. Heffer and Sons, Cambridge, pp. $164 \mathrm{ff}$.

Hellweg, G. (1954). A.M.A. Arch. Path., 57, 425.

Huseby, R. A., and Boyden, E. A. (1941). Anat. Rec., 81, 537.

Huzly, A. (1942). Anat. Anz., 93, 1.

Jaffe, K. (1921). Z. anat. entwickl. Gesch., 60, 411.

Keith, J. D., Rowe, R. D., and Vlad, P. (1958). Heart Disease in Infancy and Childhood. The Macmillan Company, New York, pp. 338 and 340.

Laurence, K. M., and Brown, R. J. K. (1960). Brit. Heart J., 22, 295.

Lawrence, T. W. P., and Nabarro, D. (1901-02). J. Anat., Lond., 36, 63.

Miller, A. J. (1925). Amer. J. Path., 1, 467.

Nabarro, D. (1902-03). J. Anat. Physiol., 37, 382.

Rogers, H. M., and Edwards, J. E. (1948). Amer. Heart J., 36, 28.

Schelenz, C. (1909). Berl. klin. Wschr., 1, 788 and 840.

Wakai, C. S., and Edwards, J. E. (1958). Amer. Heart J., 56, 779. 